\title{
Microvascular tissue as a platform technology to modify the local microenvironment and influence the healing cascade
}

\author{
Marek Dobke*,1, Dale R Peterson², Ralph-Heiko Mattern², Douglas M Arm² iD \& William \\ $\mathrm{W} \mathrm{Li} \mathrm{i}^{3}$ \\ ${ }^{1}$ Division of Plastic Surgery, Department of Surgery, University of California San Diego, 200 West Arbor Drive, San Diego, CA \\ 92103, USA \\ ${ }^{2}$ MicroVascular Tissues, Inc., San Diego, CA 92121, USA \\ ${ }^{3}$ The Angiogenesis Foundation, Cambridge, MA 02142, USA \\ *Author for correspondence: mdobke@health.ucsd.edu
}

\begin{abstract}
Aims: Profiling of microvascular tissue allows identification of components that stimulate wound healing. Here we study those elements for biological effect and establish clinical proof-of-concept using a microvascular tissue graft $\left(\mathrm{mVASC}^{\circledR}\right)$ in chronic refractory wounds. Methods: mVASC was characterized for tissue fragments and protein composition, evaluated for angiogenic potential in preclinical models, and applied clinically to a series of nonhealing wounds with compromised vascularity of different etiologies. Results: mVASC increased endothelial cell migration in vitro and angiogenesis in mouse ingrowth and hindlimb ischemia models. Clinically, mVASC stimulated wound neovascularization, granulation and epithelialization, and complete and durable healing. Conclusion: Microvascular tissue contains elements relevant to tissue repair and can be clinically applied to enable or accelerate the closure of challenging wounds.
\end{abstract}

First draft submitted: 28 October 2019; Accepted for publication: 2 March 2020; Published online: 31 March 2020

Keywords: angiogenesis $\bullet$ cell therapy $\bullet$ growth factors $\bullet$ microenvironment $\bullet$ microvascular tissue $\bullet$ radiation lesion - revascularization • sarcoma resection • wound healing

Nonhealing wounds affect up to 6 million people in the USA, resulting in healthcare expenditures exceeding US\$3 billion per year [1-3]. During normal healing, angiogenesis leads to revascularization of tissue and the establishment of a functioning microcirculation to deliver oxygen, nutrients and paracrine factors required for proper tissue repair [4]. In chronic or delayed-healing wounds, however, this process can be severely compromised. Such problematic wounds can develop for an array of reasons, such as venous stasis, ischemia, unrelieved pressure, chemotherapy and radiation treatment. Conventional clinical treatment for chronic wounds include sharp debridement, offloading of the wound to relieve pressure, treatment of infection and surgical revascularization or angioplasty. When these measures have failed, additional therapies such as negative pressure wound therapy or hyperbaric oxygen chambers have been utilized [5]. Effective therapy for patient with severe illnesses, such as advanced diabetes, critical limb ischemia, morbid obesity or cancer, who have chronic wounds can be challenging, due to underlying systemic disease and compromised tissue surrounding the wound.

A growing number of advanced treatments for wound care involve biological modalities that act on the cellular level. The earliest of these was autologous platelet-rich plasma, where patients' blood is drawn and their platelets isolated, concentrated and administered to the wound bed. While clinical benefits have been observed, and plateletrich plasma is widely used, a meta-analysis concluded that the effect on wound healing is relatively small [6]. Other cellular interventions have been studied, including autologous bone marrow-derived stem cells, stromal vascular fraction (SVF) from adipose tissue and other cellular sources, for treating nonhealing wounds in the setting of 
chronic radiation injury, severe arterial-venous leg ulceration, and with other complex co-morbidities including wounds at amputation stumps [7-10].

Allogeneic skin graft substitutes containing viable cells such as Apligraf ${ }^{\circledR}$ and Dermagraft ${ }^{\circledR}$ (Organogenesis, MA, USA) have been used in wound healing for decades [11]. Skin TE ${ }^{\circledR}$ (Polarity TE, UT, USA) is derived from a patient's own skin tissue [12]. Conditioned media containing expressed factors but no viable cells obtained from adipose tissue may induce angiogenesis and cell proliferation [13]. A topical recombinant growth factor, becaplermin (Regranex ${ }^{\circledR}$, Ortho-McNeil, NJ, USA) stimulates wound healing by promoting angiogenesis, stem cell recruitment and epithelialization. In addition, recent clinical studies with amniotic membranes, human amnion allografts or processed amnion products such as Epifix ${ }^{\circledR}$ (MiMedx, GA, USA) have demonstrated efficacy for treating diabetic foot ulcers and other healing problems [14,15]. However, there are still many complex chronic wounds that do not heal despite conventional and current advanced treatments, leading to eventual amputation.

Insufficient vascularity and a resulting senescent microenvironment are two important common denominators in failed healing [4,16-18]. Microvascular tissue is generated by the process of angiogenesis, which results in the development of new blood vessels containing vascular endothelial cells, pericytes, smooth muscle cells, fibroblasts and progenitor cells $[19,20]$. Extracellular matrix (ECM) proteins, comprising several different matrices, also are intrinsic and extrinsic to the structure of blood vessels. The endothelial and other cells within a newly formed vessel not only support improved perfusion, but also release paracrine functions that lead to cellular stimulation surrounding blood vessels within the wound bed [21].

Angiogenesis is regulated by signaling cascades activated by multiple cytokines released following local injury. Endothelial cells, pericytes and MSCs become activated, and establish a regenerative microenvironment by undergoing a sequence of growth steps and by secreting bioactive molecules that regulate local immune responses, including inflammation [22,23]. Within a few hours following injury, new capillaries begin sprouting from pre-existing venules and begin self-organizing into a proliferating microvascular tissue network in the wound bed [24,25]. As healing proceeds, vascular maturation leads to a hierarchical organization of the new blood vessels, with recruitment of smooth muscle cells and pericytes that stabilize blood vessel walls. The ability for vessels to spontaneously form networks suggests that isolated microvessel fragments have the intrinsic ability to participate in self-assembly in the context of injury [26,27]. Microvascular tissue fragments from allogeneic cell and tissue sources have been shown to exhibit high proliferation rates and pro-angiogenic effects [26,28].

To demonstrate the ability of microvascular tissue fragments to participate in wound healing, in vitro and in vivo studies were conducted using microvascular tissue graft (mVASC), a novel allogeneic product derived from human tissue. Clinical proof-of-concept for mVASCs efficacy in wound healing was demonstrated in three cases of complex, nonhealing wounds.

\section{Preclinical materials \& methods mVASC preparation}

The mVASC (MicroVascular Tissues, Inc., CA, USA) structural allograft technology consists of aseptically processed and lyophilized allogeneic microvascular tissue derived from donated human tissue and contains vessels, ECM, and associated nonviable stem and regenerative cell fragments. While microvascular tissue is located within and can be harvested from nearly every tissue source in the body, the mVASC used in the studies described was isolated from human adipose tissue, obtained and processed in accordance with US FDA requirements for banked human tissue (21 CFR Parts 1270 and 1271), and in compliance with the American Association of Tissue Banks protocols. Donors for mVASC were younger than 65 years of age and could not have a diagnosis of diabetes.

Briefly, microvascular tissue harvested from donor cadavers is isolated through a minimally manipulated process involving cutting, cleaning, extracting, concentrating, lyophilizing and sterilizing the tissue. This proprietary mVASC manufacturing process is intended to eliminate the proliferative ability of all cells within the allogeneic microvascular graft but generally retain the cellular structure and functioning biologic elements of the tissue.

The resultant tissue graft (Figure 1) is stable at room temperature for more than 4 years. The final mVASC product contains a consistent amount of the lyophilized structural mVASC, which can be removed from the vial and topically applied directly to a wound site as dry powder or may be reconstituted with sterile water-for-injection per its instructions for use. In accordance with human cellular and tissue-based product (HCT/P) regulations, the mVASC product is intended for delivery to tissue sites that require repair, reconstruction, replacement or supplementation of microvascular tissue. 
Figure 1. A vial of microvascular tissue graft (mVASC $\left.{ }^{\circledR}\right)$ showing the lyophilized, sterilized cake containing microvascular tissue fragments.

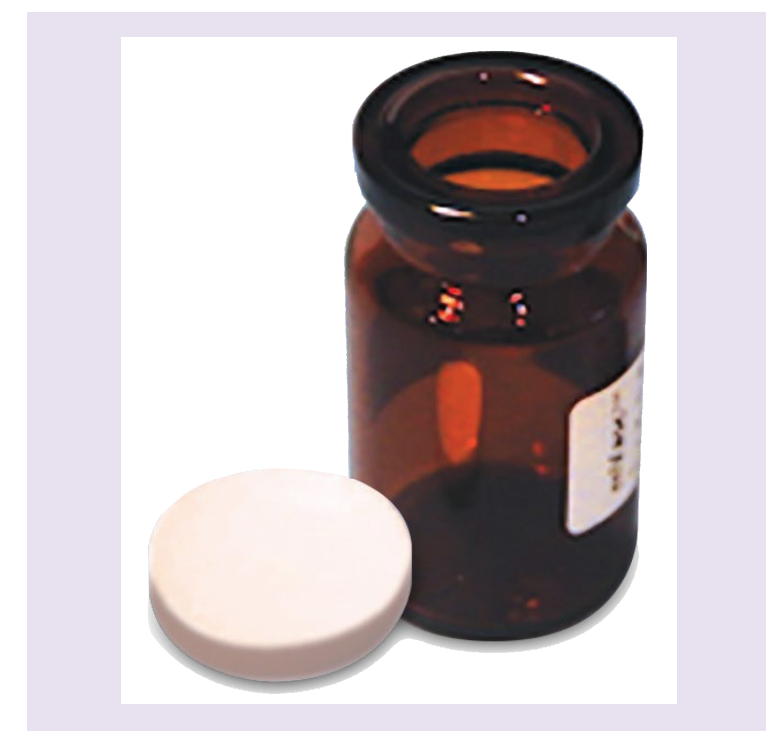

Characterization - cell counts \& viability, phenotype, structure \& proliferative capacity

Both the in-process freshly isolated microvascular tissue and the finished lyophilized and sterilized mVASC microvascular tissue product were assayed for cell counts using a hemocytometer after staining with 4',6-diamidino2-phenylindole (DAPI). Trypan Blue dye exclusion was utilized to determine viability. Three vials of mVASC were reconstituted with $1 \mathrm{ml}$ of sterile, ultrafiltered deionized water and three $1 \mathrm{ml}$ aliquots of freshly isolated microvascular cells were tested. Each $50 \mu \mathrm{l}$ of mVASC suspension were combined with $445 \mu \mathrm{l}$ of Trypan Blue and $5 \mu \mathrm{l}$ of DAPI and incubated for $5 \mathrm{~min}$. A total of $10 \mu \mathrm{l}$ of the DAPI-labeled cells or mVASC suspensions were loaded on to a hemocytometer and then imaged on an EVOS fluorescence microscope (Advanced Microscopy Group, WA, USA) using a blue fluorescence filter $(\mathrm{Ex}=357 \mathrm{~nm} ; \mathrm{Em}=447 \mathrm{~nm})$. Representative images of each sample were taken and two individual counts were performed on the EVOS. Each field (total of four) of the hemocytometer chamber was counted for DAPI-stained nuclei and any fragments that are Trypan Blue negative. Values were transferred to Microsoft Excel spreadsheets for tabulation and statistical analyses. In addition, to demonstrate lot-to-lot consistency of the mVASC tissue graft preparation, microvascular fragment counts were made on the final sterilized product on four consecutive lots using the same methodology.

In an effort to demonstrate that despite being subjected to lyophilization and sterilization, the cell membranes were relatively intact and functional, and that the composition of the preparation had not been significantly altered by the processing, both freshly isolated cells and re-suspended mVASC from two donor lots were further characterized by immunophenotyping. CD31 (endothelial cell adhesion marker); CD34 (hematopoietic and other progenitor or stem cells, pericytes and some endothelial cells); CD44 (an adhesion glycoprotein found on stromal cells and MSCs); CD45 (leukocyte common antigen found on all hematopoietic cells except erythrocytes and platelets); CD90 (variable: MSCs, immature hematopoietic stem cells, some neurons, connective tissue, activated endothelial cells, fibroblasts, keratinocyte stem cells) and collagen type IV (biomarker of microvascular ECM) were all assessed by fluorescence microscopy. By labeling with collagen Type IV and viewing under fluorescence microscopy, structural integrity of mVASC was examined. All antibodies were procured from commercial vendors (R\&D Systems, Inc. [MN, USA] or Abcam plc [MA, USA]), and were diluted 1:100 by volume for the assay. To confirm the lack of viability within mVASC, a standard colony forming unit-fibroblast assay was used to evaluate the proliferative capacity of both freshly isolated cells and mVASC.

\section{Characterization - biomarker assessment}

The mVASC tissue product was assessed by Luminex bead-based multiplexing sandwich immunoassays for the presence of 45 biomarkers associated with various functional activities including vascularization, tissue healing, inflammation, immunomodulation and nerve repair. Briefly, a suspension of microsphere sets was encoded by fluorescent dyes to individualize them to a specific individual biomarker assay and placed into a 96-well microplate. The presence and amounts of the specified biomarkers in duplicate samples of four manufacturing lots of resuspended mVASC were determined quantitatively with a Luminex bead reader, with the magnitude of the 
signal in direct proportion to the amount of analyte bound to the microspheres. Biomarker concentrations were determined by utilizing the median fluorescence intensity readings of calibration curves.

Angiogenic potential - human umbilical vascular endothelial cell migration assay

mVASC was evaluated in an in vitro human umbilical vascular endothelial cell (HUVEC) migration assay to determine its ability to preferentially draw endothelial cells across an $8-\mu \mathrm{m}$ pore-size membrane over a 48 -h incubation period. DiI-labeled HUVECs $\left(10^{4}\right.$ cells/96-Transwell ${ }^{\circledR}$ insert) in $75 \mu$ of ZTM:A2 ${ }^{\text {TM }}$ media (INCELL, TX, USA) were placed in the upper chamber of a Transwell plate. M3:DC ${ }^{T M}$ base media served as the negative control, while the positive control was M3:DC media + EGF. mVASC-induced migration was compared with the positive and negative controls, as well as freshly isolated SVF from human adipose tissue. mVASC samples were suspended in $1 \mathrm{ml}$ of ultrafiltered deionized water and all test articles were plated into the bottom of the 96-well Transwell plates in duplicate at $150 \mu \mathrm{l}$ per well. The plates were incubated for $48 \mathrm{~h}$ and were read on a Gemini plate reader (Molecular Devices, CA, USA) for fluorescence $(E x=533 \mathrm{~nm}$ and $\mathrm{Em}=570 \mathrm{~nm}$ ) from the top to estimate the amount of DiI, which reflected the relative numbers of HUVECs stimulated to migrate into the lower chamber of the plate (expressed as 'relative fluorescence units').

\section{Angiogenic potential - Matrigel ingrowth assay}

An established severe combined immunodeficiency (SCID) mouse Matrigel model used to study blood vessel ingrowth was employed to compare saline control versus two doses of mVASC. In an IACUC-approved protocol, immunodeficient male NOD SCID mice (14-16 weeks of age) were implanted with two $0.5-\mathrm{ml}$ Matrigel plugs containing either high concentration of mVASC $\left(0.5 \times 10^{6}\right)$ fragments, low concentration of mVASC $\left(0.5 \times 10^{5}\right)$ fragments or saline control. In this model, all Matrigel plugs (including the negative control) were also supplemented with $200 \mathrm{ng} / \mathrm{ml}$ bFGF, to provide a baseline with some (but suboptimal) angiogenesis to both verify the assay was functioning and enable observation of a possible inhibitory mVASC effect. The high concentration mVASC plugs were prepared by directly dissolving one full vial containing $0.5 \times 10^{6}$ fragments of lyophilized mVASC into Matrigel supplemented with $200 \mathrm{ng} / \mathrm{ml} \mathrm{bFGF}$. Low concentration plugs were prepared by diluting high-dose suspensions 1:10 into additional bFGF-supplemented Matrigel. Animals were anesthetized with $2 \%$ isoflurane and kept on a heated pad to maintain body temperature. The animals were then shaved and depilated locally before injection, to allow for better visualization during the procedure. The Matrigel plugs were implanted subcutaneously into the right and left dorsal flanks, using syringes equipped with $20 \mathrm{~g}$ needles. After 2 weeks, animals were sacrificed by cervical dislocation. Matrigel plugs were excised with adhering tissue, fixed in $10 \%$ neutral buffered formalin and stored in $70 \%$ ethanol for further analysis.

After fixation, plugs were paraffin-embedded and sectioned for immunofluorescence assessment. To analyze vessel formation, sections were stained with antibodies against surface markers for endothelial cells (rat monoclonal anti-mouse CD31, Dianova, Hamburg, Germany; sheep polyclonal anti-human CD31, R\&D Systems, MN, USA). Primary antibodies were visualized with fluorophore-conjugated secondary antibodies (Dylight 594 anti-rat and Dylight 488 anti-sheep, both Abcam, MA, USA). Images were captured using a Nikon Eclipse E1000M microscope (Nikon, CA, USA). Multiple sections spanning the whole plug were obtained, stained and 84 high-power fields from 21 different sections were manually analyzed for CD31 positive tube formation using FIJI software (NIH, MA, USA). The newly formed vessels were quantified and categorized into three groups: (1) small tubes with a diameter of $<10 \mu \mathrm{m}$ without a visible lumen; (2) medium tubes with a visible lumen and (3) large tubes with a diameter of more than $10 \mu \mathrm{m}$ with a visible lumen and visible erythrocytes in the lumen.

\section{Angiogenic potential - hindlimb ischemia study}

An SCID mouse hindlimb ischemia model was used to study the effect of mVASC on ischemia-reperfusion following the ligation and transection of the femoral artery in one hindlimb. Hindlimb ischemia was surgically induced into 8 NOD SCID male mice (Jackson Labs, ME, USA, 13 weeks old) as described in previous publications using an IACUC-approved protocol [29,30]. After induction of anesthesia with $2 \%$ isoflurane and $1 \mathrm{l} / \mathrm{min}$ flow rate of $\mathrm{O}_{2}$, the femoral artery of the left leg was dissected and separated from the femoral vein and nerve. A strand of 7-0 silk sutures was passed underneath the proximal end of the femoral artery and the artery was occluded. The distal femoral artery was occluded by the same procedure and the segment of the femoral artery between the knots was excised. After surgery, animals were placed on top of a draped heated pad in a recovery cage and monitored until awake. 
Figure 2. Fluorescent micrographic image of microvascular tissue graft showing microvascular tissue fragments with intact vessel structure, as indicated by positive staining for type IV collagen. The microvascular structure serves as conduits for revascularization.

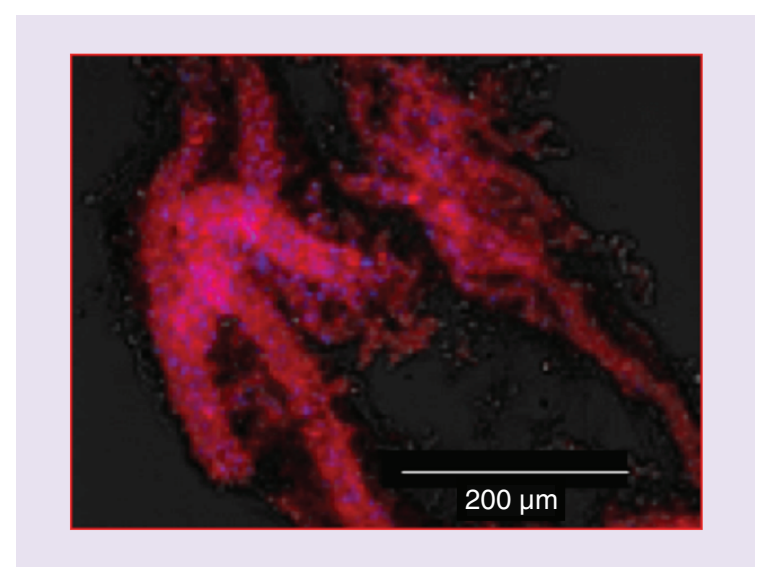

Table 1. Comparison of cell (or cell fragment) concentrations and viability based on 4',6-diamidino-2-phenylindole staining and \% trypan blue exclusion between freshly isolated cells and microvascular tissue graft. The lyophilization and radiation sterilization processes rendered the cell fragments nonviable, although more than $87 \%$ of the cellular fragments were conserved in the final microvascular tissue graft formulation.

\begin{tabular}{|lcc|}
\hline Assay & Isolated cells & mVASC \\
\hline \# (DAPI)/gram tissue & $4.8 \pm 0.4 \times 10^{5}$ & $4.3 \pm 0.3 \times 10^{5}$ \\
\hline Viability (trypan blue) & $83 \pm 7 \%$ & Below sensitivity \\
\hline DAPI: 4',6-diamidino-2-phenylindole; mVASC: Microvascular tissue graft. & \\
\hline
\end{tabular}

The 8 animals were divided into two groups of 4 mice each. Animals in the treatment group were injected with $100 \mu \mathrm{l}$ of mVASC freshly resuspended in sterile deionized water on days 0,3 and 7 . A total of $70 \mu$ was injected into the left adductor muscle and $30 \mu \mathrm{l}$ into the left gastrocnemius muscle of the ischemic limb. The 100- $\mu$ l injections contained $1.7 \times 10^{5}$ mVASC tissue fragments, resulting in a cumulative total of $5 \times 10^{5}$ microvascular fragments delivered the animals over the three injections. Animals in the control group received 100- $\mu$ l injections of saline at the same time points.

Measurement of tissue perfusion was made on days 0, 7 and 14 using Laser Doppler Imaging under anesthesia. The animals were then placed on a heat plate to adjust the body temperature to $37.5^{\circ} \mathrm{C}$, and respiratory rate constantly monitored for signs of stress and discomfort from overheating or insufficient anesthesia. The animals were placed in a supine position at the measurement platform and microvascular perfusion was measured via laser Doppler scans of the entire lower body over 3-min cycles. Relative mean perfusion rate was calculated by quantifying the blood flow in the ischemic limb in relation to the nonischemic control limb.

\section{Animal care approvals}

Approvals for the Matrigel ingrowth and hindlimb ischemia study protocols were obtained through the Association for Assessment and Accreditation of Laboratory Animal Care-accredited Stanford University Administrative Panel on Laboratory Animal Care, and all experiments were conducted in compliance with the Administrative Panel on Laboratory Animal Care approvals.

\section{Preclinical results}

Characterization - structure, cell counts \& viability, phenotype \& proliferative capacity

A fluorescent micrographic image of mVASC shows microvascular tissue fragments with intact vessel structure, as indicated by positive staining for type IV collagen (Figure 2).

As shown in Table 1, using DAPI staining and \% trypan blue dye exclusion microscopy assays 484,000 nucleated cells per gram of tissue were counted in the output immediately following isolation of cells and cell aggregates from the microvascular tissue, with $83 \%$ viability. While $87.6 \%$ of the cell and tissue fragments $(429,000$ per gram) were conserved in the final mVASC formulation, the lyophilization and radiation sterilization processes rendered the cell fragments nonviable, below the sensitivity of the trypan blue assay. The mean number of microvascular fragments 


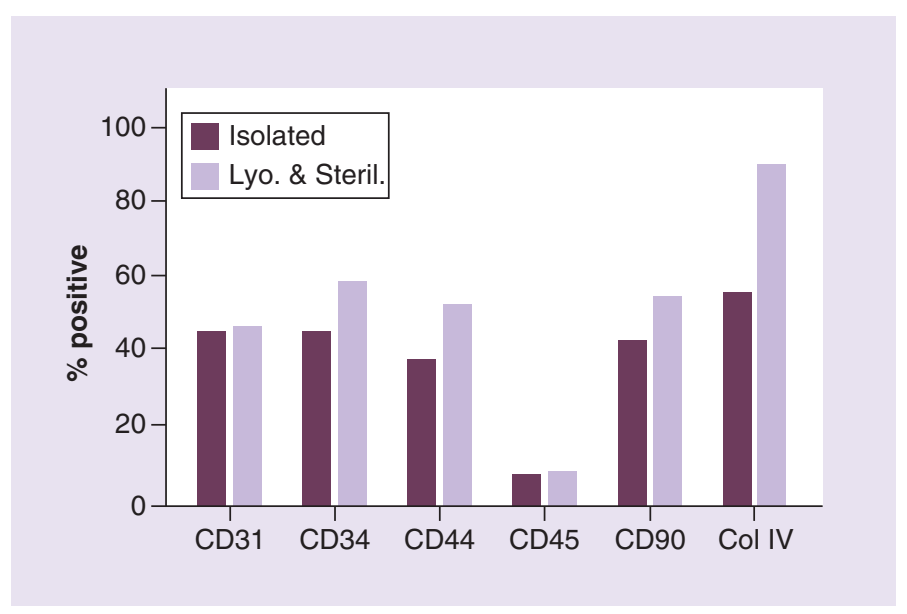

Figure 3. Biomarker characterization of mVASC. Immunophenotypingresults of selected biomarkers in mVASC show conservation throughout processing. Lyo.: Lyophilized; mVASC: Microvascular tissue graft; Steril.: Sterilized.

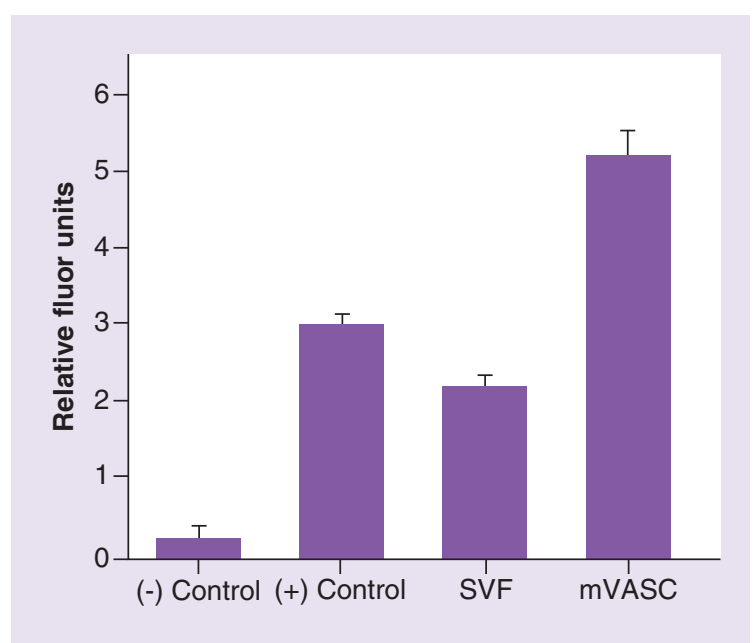

Figure 4. Microvascular tissue graft-induced human umbilical vascular endothelial cell migration in vitro was compared with media $\pm E G F$ controls as well as freshly isolated stromal vascular fraction from adipose tissue. mVASC, SVF and the positive control with EGF all significantly induced migration of HUVEC compared with the media alone negative control. The lyophilized and sterilized mVASC outperformed the positive control and live cells by 72 and $134 \%$, respectively. HUVEC: Human umbilical vascular endothelial cell; mVASC: Microvascular tissue graft; SFV: Stromal vascular fraction.

in four consecutive lots was $1.206 \pm 0.276 \times 10^{6}$ per vial, a coefficient of variance of $22.9 \%$, demonstrating that the processing controls utilized during mVASC production resulted in a consistent final product.

Figure 3 shows immunophenotyping data for selected biomarkers. Of note, while all biomarkers were conserved through the mVASC processing, after sterilization some biomarkers (CD34, CD44, CD90 and collagen type IV) actually became more prevalent.

No colony forming unit-fibroblast formed from the sterilized mVASC preparations, indicating that the cell fragments within mVASC lost all the ability to proliferate. Freshly isolated viable microvascular tissue/cell aggregates did form colonies as expected (data not shown).

\section{Characterization - biomarker assessment}

Multiplex ELISA confirmed the presence of numerous factors associated with various functional activities including vascularization, tissue healing, inflammation, immunomodulation and nerve repair. Table 2 documents the presence of the growth factors, cytokines and chemokines found in the mVASC tissue product, and indicates the biological function of each biomarker. In Figure 3B, concentrations of select biomarkers as determined by multiplex ELISA analysis from four unique lots of mVASC demonstrate consistency across donors.

\section{Angiogenic potential - HUVEC migration assay}

mVASC, SVF and the positive control with EGF all significantly induced migration of HUVEC over a 48-h incubation period compared with the media-alone negative control (Figure 4). The lyophilized and sterilized mVASC outperformed the positive control and live cells by 72 and $134 \%$, respectively, indicating its high potency to induce microvascular repair and reconstruction. 
Table 2. Multiplex ELISA analysis confirmed presence of numerous factors associated with relevant functional activities including vascularization, tissue healing, inflammation, immunomodulation and nerve repair. Relative prevalence of each factor is indicated by present $(+)$, abundant $(++)$ and not significantly present (-).

\begin{tabular}{|c|c|c|c|c|c|}
\hline Factor & Present & 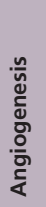 & 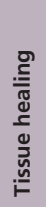 & 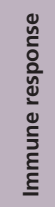 & $\begin{array}{l}\frac{n}{y} \\
\frac{1}{c} \\
0 \\
\frac{0}{2} \\
\frac{0}{3} \\
\frac{d}{z}\end{array}$ \\
\hline Angiogenin & ++ & $\checkmark$ & & & \\
\hline Angiopoietin-2 & ++ & $\checkmark$ & & & \\
\hline BDNF & ++ & $\checkmark$ & & & $\checkmark$ \\
\hline BMP-2 & + & & $\checkmark$ & & \\
\hline BMP-4 & + & & $\checkmark$ & & $\checkmark$ \\
\hline BMP-12 & - & & $\checkmark$ & & 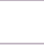 \\
\hline BMP-13 & ++ & & $\checkmark$ & & $\checkmark$ \\
\hline CNTF & - & & & & $\checkmark$ \\
\hline EGF & + & & $\checkmark$ & & \\
\hline FGF-1 & ++ & $\checkmark$ & $\checkmark$ & & $\checkmark$ \\
\hline FGF-2 & ++ & $\checkmark$ & $\checkmark$ & & $\checkmark$ \\
\hline FGF-4 & - & & $\checkmark$ & & \\
\hline FGF-7 (KGF) & - & & $\checkmark$ & & \\
\hline FGF-18 & - & $\checkmark$ & & & \\
\hline G-CSF & ++ & & $\checkmark$ & $\checkmark$ & $\checkmark$ \\
\hline GDNF & + & & $\checkmark$ & & $\checkmark$ \\
\hline GM-CSF & + & & & $\checkmark$ & \\
\hline HGF & ++ & $\checkmark$ & $\checkmark$ & & \\
\hline ICAM & ++ & & & $\checkmark$ & \\
\hline IL-1 ra & ++ & & & $\checkmark$ & \\
\hline IL-1 $1 \alpha$ & + & & & $\checkmark$ & \\
\hline IL-4 & + & & & $\checkmark$ & \\
\hline IL-6 & + & & & $\checkmark$ & \\
\hline IL-8 & ++ & $\checkmark$ & & $\checkmark$ & \\
\hline IL-10 & + & & & $\checkmark$ & \\
\hline INF- $\gamma$ & + & & & $\checkmark$ & \\
\hline MCP-1 & ++ & & & $\checkmark$ & \\
\hline MIP-1 $1 \alpha$ & + & & & $\checkmark$ & \\
\hline MIP-1 $\beta$ & + & & & $\checkmark$ & \\
\hline MMP-1 & + & & $\checkmark$ & & \\
\hline MMP-3 & ++ & & $\checkmark$ & & \\
\hline NGF & + & $\checkmark$ & & $\checkmark$ & $\checkmark$ \\
\hline Osteopontin & ++ & & $\checkmark$ & $\checkmark$ & \\
\hline Osteoprotegerin & + & & $\checkmark$ & $\checkmark$ & \\
\hline PDGF & ++ & $\checkmark$ & $\checkmark$ & 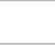 & $\checkmark$ \\
\hline PECAM-1 & ++ & $\checkmark$ & & $\checkmark$ & \\
\hline PGE-2 & ++ & & $\checkmark$ & $\checkmark$ & \\
\hline PIGF & - & $\checkmark$ & & & \\
\hline SDF-1a & ++ & $\checkmark$ & $\checkmark$ & & \\
\hline Substance $\mathrm{P}$ & - & & & $\checkmark$ & \\
\hline TGF- $\alpha$ & - & & $\checkmark$ & & \\
\hline TGF- $\beta 1$ & - & & $\checkmark$ & $\checkmark$ & \\
\hline TNF- $\alpha$ & + & & & $\checkmark$ & \\
\hline VCAM & ++ & & & $\checkmark$ & \\
\hline VEGF & ++ & $\checkmark$ & & $\checkmark$ & $\checkmark$ \\
\hline
\end{tabular}




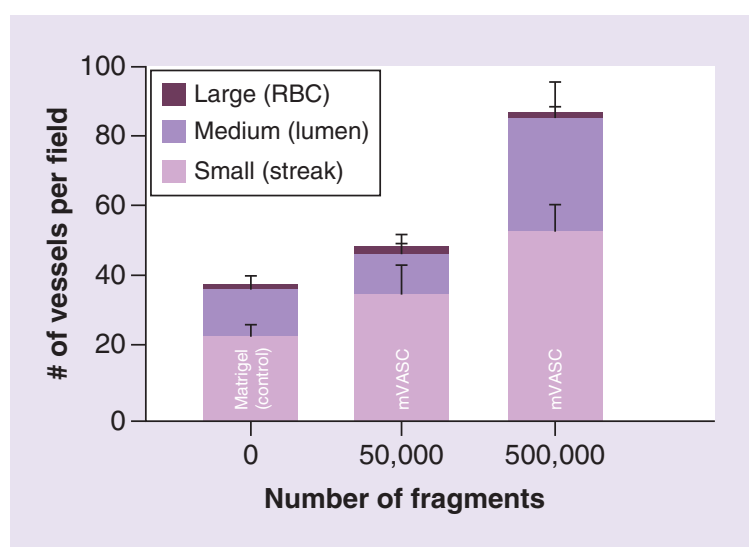

Figure 5. Quantification of blood vessel number and diameter at day 14 in an severe combined immunodeficiency mouse Matrigel angiogenesis model, comparing low and high doses of microvascular tissue graft within the Matrigel to a Matrigel-only control. Vessels were quantified and categorized into three groups: (1) Small tubes $<10 \mu \mathrm{m}$ in diameter without a visible lumen; (2) medium tubes with a visible lumen and (3) large tubes $>10 \mu \mathrm{m}$ in diameter with a visible lumen and visible erythrocytes in the lumen. RBC: Red blood cell.

Angiogenic potential - matrigel ingrowth assay

Blood vessel ingrowth in the SCID mouse Matrigel model is shown in Figure 5. Vascular ingrowth increased 33 and $139 \%$ over the control for the low and high concentrations of mVASC fragments, respectively. mVASC also led to more mature vessels, as quantified by the greater number of larger diameter vessels, predominantly due to the increase in medium-sized vessels.

\section{Angiogenic potential - hindlimb ischemia study}

Representative Doppler images and quantitative perfusion ratio results from the hindlimb ischemia study are shown in Figure 6. Complete restriction of blood flow in the injured leg following the ligation and transection of the femoral artery was achieved. At Day 7, some reperfusion was evident in both control and mVASC-treated mice, but by Day 14, the blood flow observed with immature vasculature in the control animals had stalled, with hindlimb necrosis evident. In mVASC-treated animals, the vascularization continued with nearly complete restoration of blood flow. Overall, the mean perfusion ratios at day 14 were $59 \pm 4 \%$ of the contralateral control limb for the control group and $82 \pm 8 \%$ of the contralateral limb for the mVASC group $(\mathrm{p}=0.002)$.

\section{Clinical application of mVASC}

To demonstrate clinical proof-of-concept for mVASC, human chronic wounds were treated using topical microvascular tissue. The treated patients had co-morbidities known to impair healing (hostile environment), were not candidates for surgical revascularization or skin grafting, and had two or more advanced treatments that failed to close the wound. Three wound cases are presented: postradiation injury, pharmacologically induced senescence and physical obstruction (high fluid output through a pericardial window wound). All patients provided written informed consent to receive mVASC. As mVASC is an HCT/P indicated for the repair, reconstruction, replacement or supplementation of microvascular tissue, no additional institutional approvals are required for its clinical use.

\section{Case \#1: Postsarcoma wound}

A 73-year-old male with peripheral vascular disease presented with an ulcerated recurring sarcoma of the left calf. Clinically, the tissue was ischemic and fibrotic as a result of radiation treatment preceding surgery. Following tumor resection, the wound was treated with amniotic membrane, a split thickness graft and negative pressure wound therapy but wound dehiscence occurred. To stimulate wound neovascularization, mVASC was topically applied following surgical debridement. The site was covered with Adaptic ${ }^{T M}$ (Acelity, TX, USA) nonadherent dry dressing and a tubular net bandage ( $\mathrm{TG}^{\circledR}$ Fix, Lohmann \& Rauscher, Rengsdorf, Germany) to stabilize the underlying dressings. At each follow-up visit (every 4-8 days) the wound was lightly debrided to remove the desiccating tissue layer while taking care not to disturb the visible neovascular tissue. For the first four follow-up visits, two vials of mVASC were applied topically per visit, after which one vial was applied topically for each of the subsequent three visits.

The progression of the wound is shown in Figure 7. Seven days after initial treatment, there was notable improvement in vascularity and granulation. Eleven days after initial treatment epithelialization was evident, and increased vascular formation was observed around the entire wound bed. A continued absence of granulation of the upper quadrant was apparent due to fibrotic tissue. Nine days after the start of mVASC treatments, significant 


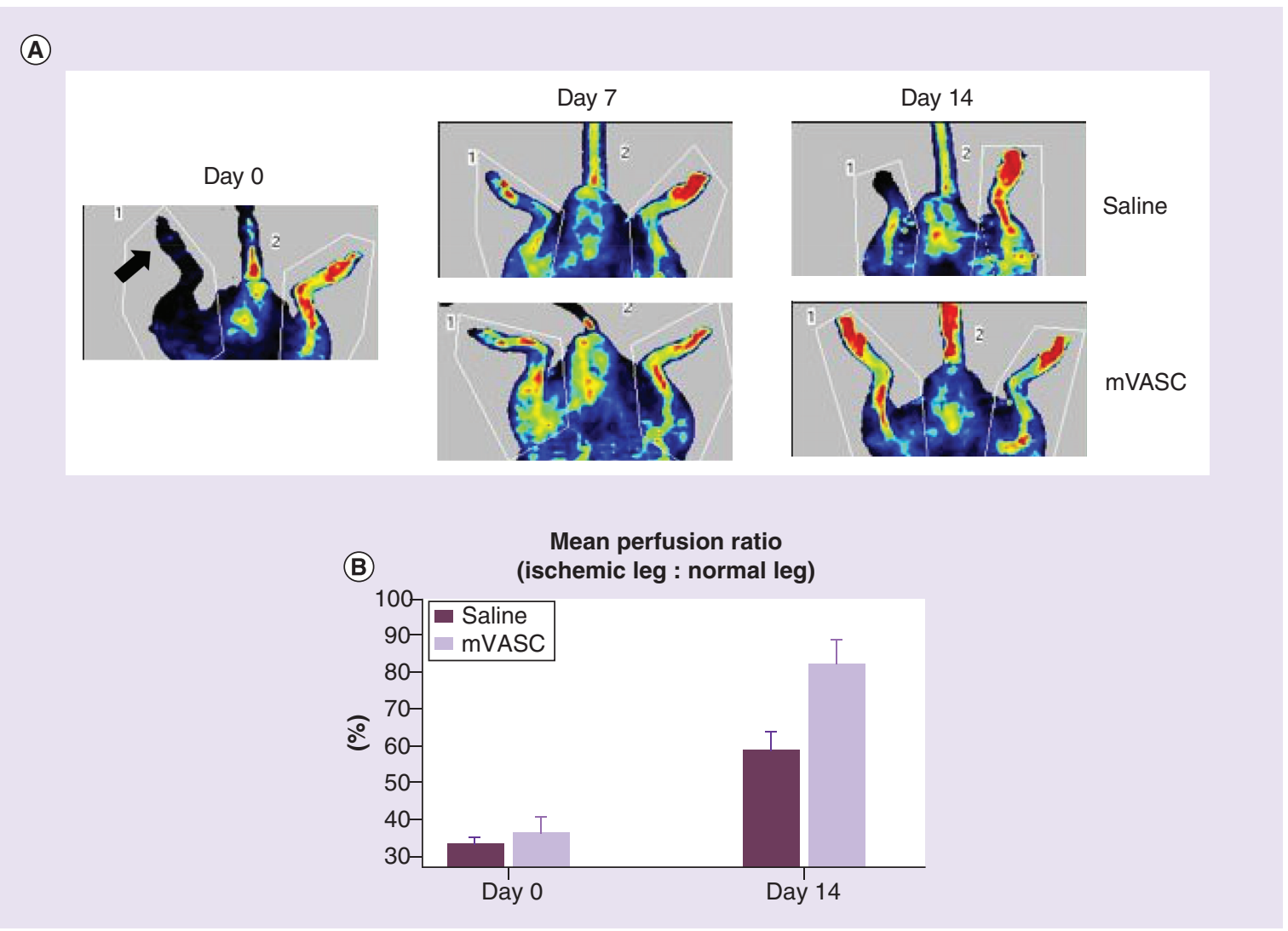

Figure 6. Effects of mVASC in hindlimb ischemia.(A) Representative Laser Doppler images of severe combined immunodeficiency mice following creation of hindlimb ischemic injury in one leg. On Day 0 , the decrease in blood flow in the injured leg (arrow) is evident. By Day 7, some early angiogenesis is evident in both control and mVASC-treated mice. By Day 14, however, the blood flow observed with immature vasculature in the control animals has been resorbed and tissue has necrosed, while in the mVASC animals the collateralization and reconstruction of the vasculature is complete. (B) Graph representing the mean perfusion ration of the ischemic leg following mVASC $(n=4)$ or saline $(n=4)$ treatment compared with the contralateral uninjured control leg. Overall, the mean perfusion ratios at day 14 were $59 \pm 4 \%$ for the control group and $82 \pm 8 \%$ for the mVASC group $(p=0.002)$. mVASC: Microvascular tissue graft.

epithelialization and development of organized tissue around the perimeter was evident. There was contiguous revascularization with substantial remodeling of the wound bed and epidermal closure of the lower quadrant of the crescent-shaped wound. At 26 days, the defect demonstrated good epithelial ingrowth, and the wound had decreased from $7 \mathrm{~cm}$ at the time of initial mVASC treatment to $4.5 \mathrm{~cm}$. By 35 days postinitial treatment, more than $50 \%$ of the defect was healed, and the relative amount of vascularized tissue to fibrotic tissue continued to increase.

By day 47, practically no fibrotic tissue was observed within the remaining nonepithelialized portion of the wound, and by day 54 , well over $75 \%$ of the crescent peripheral to the skin graft defect was filled with tissue with mature epidermis at the surface. At day 61, the wound was completely healed. Six months later the graft remained stable, wound healing was durable and the skin had the appearance, pliability and other characteristics of surrounding, native skin (Figure $7 \mathrm{~K}$ ).

\section{Case \#2: Nonhealing shin wound following Mohs' procedure}

A 76-year-old female presented with a nonhealing shin wound 3 months after a Mohs' procedure for basal cell carcinoma of the lower leg skin. The patient had also been treated with methotrexate for rheumatoid arthritis and prednisone for more than 10 years, and had no previous history of nonhealing wounds. Conventional wound treatment along with weekly application of dehydrated amnion/chorion membrane failed to decrease the size of the wound, induce epithelialization from the wound edges or stimulate healing by a contraction of this relatively 

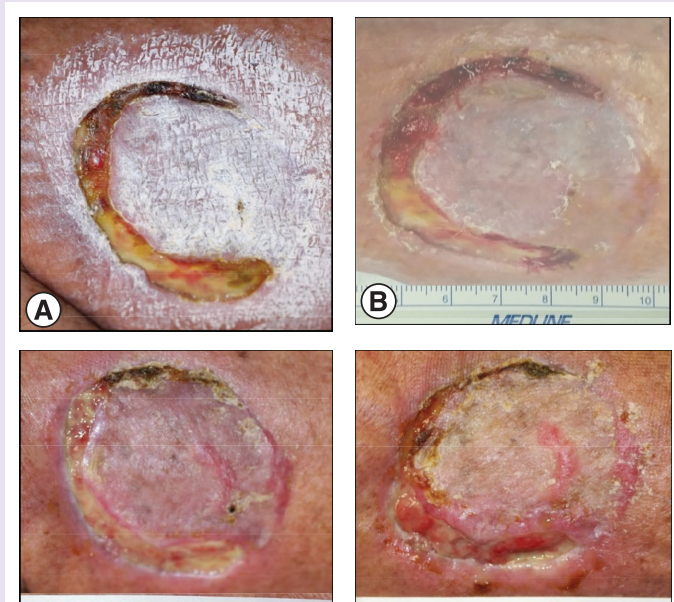

(E)

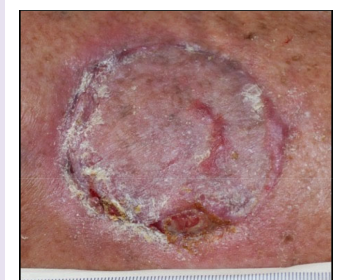

(I)
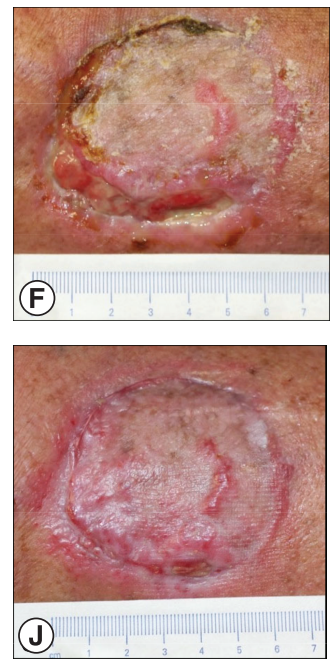
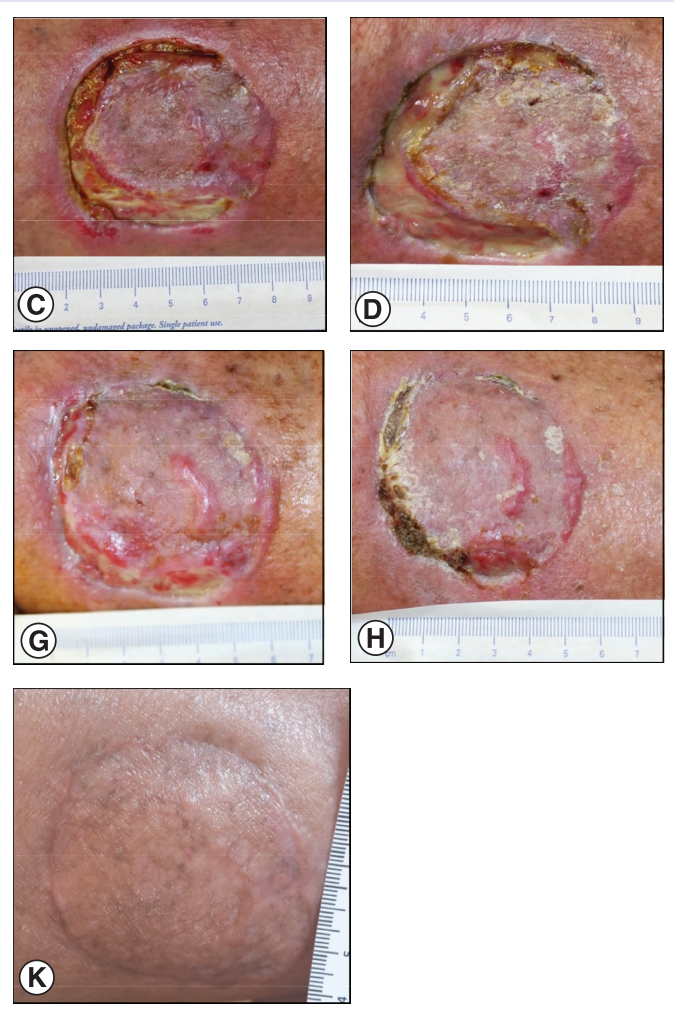

Figure 7. Progression of postsarcoma senescent wound reconstruction. Wound was treated weekly with two vials of mVASC for 5 weeks (B-F), then with one vial of mVASC for approximately three additional weeks (G-I). Unless specified otherwise, time points indicated for each figure are days after initial mVASC treatment as follows: (A) upon initial presentation; (B) after initial debridement, immediately prior to initial mVASC treatment; (C) 7 days; (D) 11 days; (E) 19 days; (F) 26 days; (G) 35 days; (H) 47 days; (I) 54 days; (J) 61 days after initial mVASC treatment - no mVASC re-application, wound considered closed; (K) 6 months follow-up; healed surface remains stable, no secondary breakdowns. Informed written consent for the publication or presentation of the case information and photos were provided by the patient.

mVASC: Microvascular tissue graft.

small defect. mVASC was then topically applied in an even distribution over the $1 \mathrm{~cm}^{2}$ wound surface following mechanical debridement to remove necrotic tissue. A nonadherent $\left(\right.$ Adaptic $\left.^{\top M}\right)$ dressing was placed over the wound, Steri-Strips ${ }^{\top M}(3 \mathrm{M}, \mathrm{MN}$, USA) were applied and covered with a standard dry dressing. The patient was instructed not to change the wound dressing or to bathe and to return weekly for assessment of wound healing (Figure 8).

One week after topical mVASC application at the initial visit, tissue granulation and progressive epithelialization was noted, and new tissue remodeling was present. At 2 weeks after the single mVASC application, there was continued revascularization and significant remodeling of the wound bed, with abundant healthy tissue both surrounding the wound site and the middle of the wound, and the wound was deemed closed. At follow-up visits 3 weeks, 4 weeks and 6 months after mVASC application, wound closure was confirmed, with continued re-epithelialization and tissue granulation evident and healthy, well-vascularized tissue surrounding the site.

\section{Case \#3: Draining pericardio-cutaneous fistula with senescent pericardial window wound following treatment of heart sarcoma}

A 50-year-old male with sarcoma of the heart who was undergoing chemotherapy and had serious co-morbidities including heart failure developed a significant effusion of pericardial fluid. A pericardial window procedure was performed. The access wound opened after layered closure and effusions were persistently draining across the chest wall. The wound dehisced and remained unhealed despite frequent change in wet to dry saline dressings over a period of 6 weeks. Due to the risk of serious infection, the patient was subsequently referred to plastic 

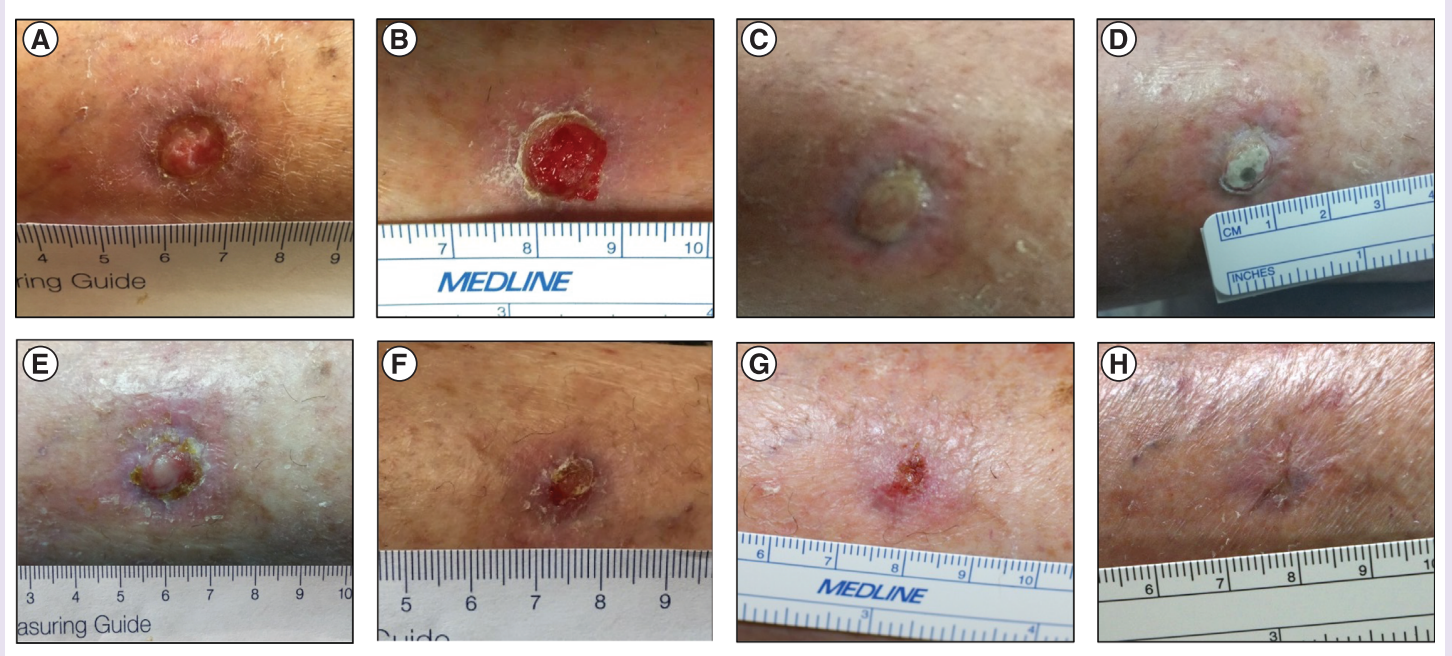

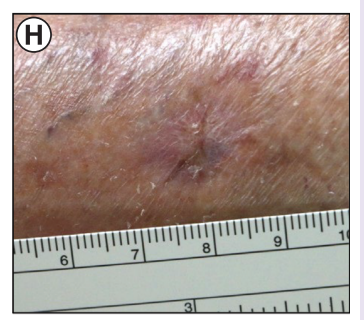

Figure 8. Progression of Mohs' shin wound healing after mVASC treatment. (A) Upon initial presentation; (B) after initial debridement, prior to mVASC application; (C) 1 week after mVASC application epithelialization and tissue granulation evident; (D) silver nitrate confirmed vascularity of new tissue 1 week after mVASC application; (E) 2 weeks after single mVASC application wound considered closed; (F) 3 weeks after mVASC application wound closure confirmed; (G) 4 weeks after mVASC application healthy, well-vascularized tissue throughout the site; (H) 6 months follow-up; healed surface stable, practically indistinguishable from surroundings. Informed written consent for the publication or presentation of the case information and photos was provided by the patient. mVASC: Microvascular tissue graft.

surgery for wound management and was determined to have a senescent wound track from the skin surface to exposed pericardial tissue. Management with topical mVASC was chosen as a noninvasive option in an attempt to promote healing in a patient whose condition precluded invasive intervention. The wound was thoroughly debrided to remove fibrinous and necrotic tissue and excise the nonvascular tissue around the wound edges, followed by mVASC application and then covered with Adaptic. Progression of wound repair is shown in Figure 9. At week 1, wound size reduction was noted, the wound lightly debrided to expose punctate bleeding at the wound bed and mVASC re-applied. By week 2, the leak had sealed and the wound had re-epithelialized and by week 3 , the wound had closed.

\section{Discussion}

The recognition that microvascular tissue may be a feasible starting source for a cell- or tissue-based therapy has been explored by both McDaniel et al. and Laschke and Menger [26,28]. In their work, the isolation and transplantation of viable adipose tissue-derived microvascular fragments demonstrated that these fragments rapidly develop into blood-perfused microvascular networks after their transfer into tissue defects. These microvascular fragments have been shown to exhibit high proliferation rates and ability to form capillary networks. The mVASC processed mVASC containing microvascular fragments with a multitude of active tissue repair factors but no viable (proliferating) cells. In addition, mVASC's microvascular structure serves as an ECM conduit for revascularization while its composition may provide benefits of angiogenic and tissue healing signaling effects, positioning it as a promising platform technology to address conditions of compromised vascularity. Donor-to-donor heterogeneity, recognized as a concern in allogeneic tissue graft products, does not appear to be a concern with mVASC based upon the consistency of microvascular fragment counts. In part, this concern is alleviated because of the normalization of the number of fragments dispensed into each mVASC vial.

Microvascular tissue supports organ development and healing throughout life, and includes cellular, matrix and cytokine elements. Endothelial cells, pericytes and smooth muscle cells are key cellular components of the microvasculature and are required for wound repair and regeneration [22,31].

The rationale for transplanting microvascular tissue, with the goal of repairing, reconstructing, replacing or supplementing the microvasculature in order to create new functional microcirculation within the local environment, is thus evident. A healthy microvascular tissue structure is critical for the function and survival of most tissues 

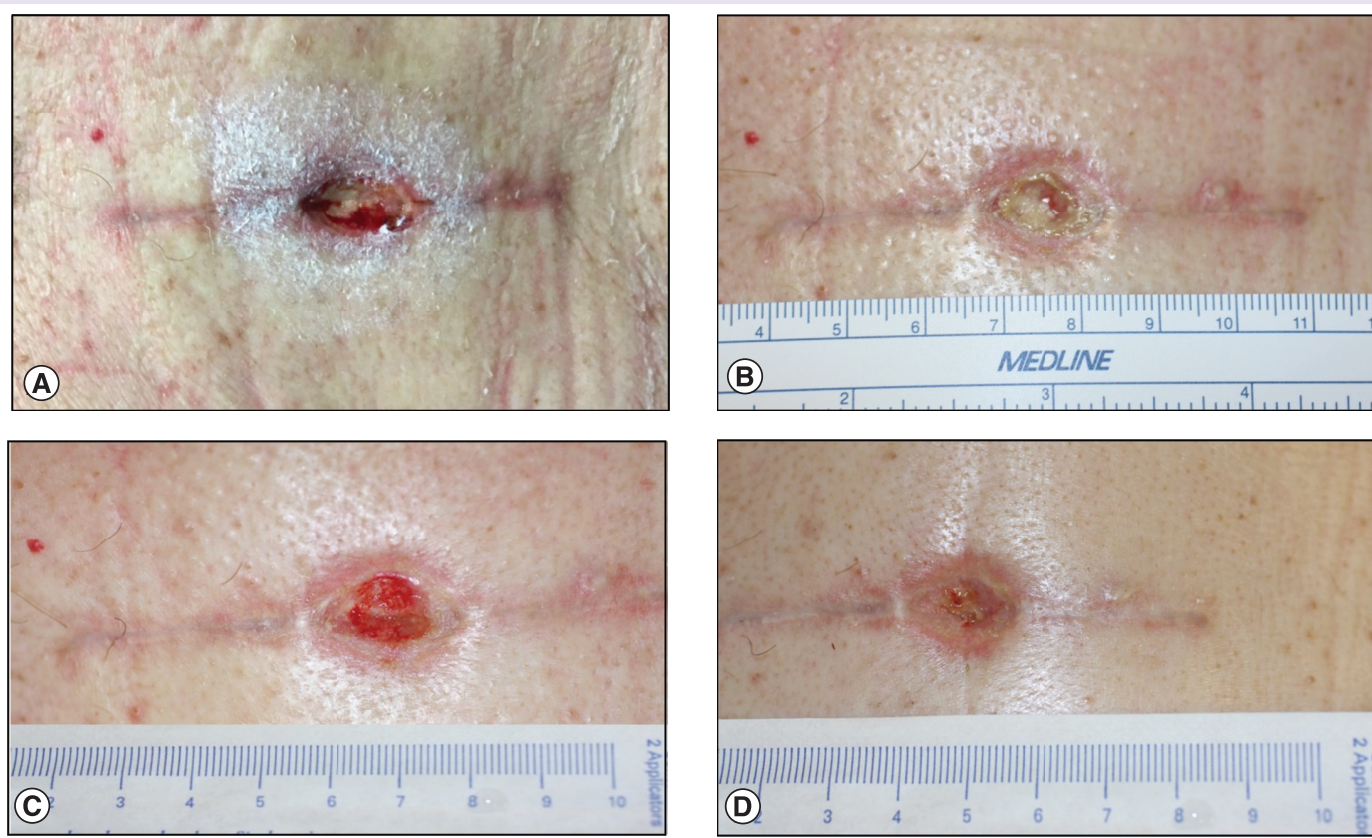

Figure 9. Progression of cardiac cancer senescent pericardial window wound. (A) Upon initial presentation, treated with 1 vial mVASC; (B) 1 week after first mVASC application, re-treated with 1 vial mVASC; (C) 2 weeks after first mVASC application and light debridement, re-treated with 1 vial mVASC; (D) 3 weeks after first mVASC application, wound considered closed. Informed written consent for the publication or presentation of the case information and photos was provided by the patient.

mVASC: Microvascular tissue graft.

and is essential for nutrient and oxygen delivery as well as removal of waste metabolites. The creation of a healthy microvascular tissue structure critically depends on the formation of new vessels (e.g., due to angiogenesis) and the integration of the new vessels into a microvascular network that meets tissue perfusion and functional demands [32].

The transplanted ECM, from the vessel basement membrane, provides physical scaffolding, mechanical stability and biochemical cues necessary for angiogenesis, tissue formation and maintenance of stability in the microenvironment [33]. The ECM is capable of exchanging information with cells and modulates a whole host of processes including development, cell migration, attachment, differentiation and repair [34], and also serves as a reservoir and modulator for growth factors [35,36].

In the presented studies, mVASC demonstrated an increase in endothelial cell migration in vitro, augmented angiogenesis in a Matrigel mouse model and significantly increased collateralization leading to restored circulation in a rat hindlimb ischemia model. In addition, Gimble et al. demonstrated that the one-time injection or topical application of mVASC to the wound site in a murine pressure ulcer model accelerated wound closure and eschar separation, raised skin temperature surrounding the site consistent with increased blood flow, upregulated factors such as $\alpha$-smooth muscle actin, VEGF and PDGF that stimulate angiogenesis and stem cell homing, accelerated disappearance of inflammatory myeloid cells $\left(\mathrm{CD} 11 \mathrm{~b}^{+}\right)$and increased the number of vascular and certain neural cell populations [37].

The question of delivery modality is an important one to consider in the context of clinical use and indication. mVASC's formulation and packaging enables its utility both reconstituted for injection or in powder form for topical application. As an injectable, it can be implanted directly into a subsurface target environment, as was done in the hindlimb ischemia model, in a way that is not feasible with the unreconstituted powder. For an exposed surface, administration of topical mVASC powder adsorbs quickly into the recipient's tissue bed following superficial debridement of the site. Gimble et al. [37] utilized both topical and injection delivery methods for treating murine skin ulcers, with enhanced healing and reduced time for closure observed for both modalities compared with controls. However, the topical application yielded slightly better results for a surface wound environment, 
and this is the approach used in a randomized controlled clinical trial evaluating mVASC for nonhealing wounds currently in progress. Further studies exploring the differences and relative benefits of these delivery methods are planned.

Inflammation plays an early role in wound healing. Local macrophages, mesenchymal stem cells (MSCs) and other cells migrate into the wound site by chemotaxis or stimulation $[5,38,39]$. The use of mVASC as a mVASC to repair, replace and reconstruct microvascular tissue appears to change the trajectory of wound healing, and clinical outcomes support the concept that mVASC enhanced the wound healing process by cutaneous tissue growth with little or no inflammation. The shortening or abolishment of the inflammatory phase of healing, with stimulation of proliferative, reparative and possibly regenerative processes through signaling of an increased production of growth factors and generation of neovascularity, appears to accelerate the healing of chronic, complex wounds. Clinical observation of the elimination of wound senescence does not necessarily imply true reversal of phenotypic cellular senescence on a molecular level. Other plausible mechanisms of action may include removal of senescent, healing inhibiting, cells via the senolytic pathway [40,41]. Additionally or alternatively, it is possible that mVASC may reactivate the hypoxia-inducible factor (known as HIF-1 $\alpha$ ) pathway, leading to amplification of local vasoactive reactions and vascularity, and thus overpowering the effect of the senescent cells' presence [42].

The mVASC product is easy to apply to wounds and is stable at room temperature. As it does not contain any viable cells, it is therefore not subject to any of the concerns that can be attributed to live cell-based products. As mVASC is an HCT/P indicated for the repair, reconstruction, replacement or supplementation of microvascular tissue, no additional institutional approvals are required for its clinical use. In all proof-of-concept cases, standard care failed to heal the wounds, and revascularization and healing only occurred following the topical application of mVASC. In addition, the ability to use an off-the-shelf tissue technology such as mVASC to successfully revascularize complex wounds in an office setting offers not only a physical benefit to compromised patients who may not be able to withstand an invasive procedure, but also may lower the cost of healing wounds by averting the need for an operative procedure and hospital admission.

Within this clinical proof-of-concept series, the application of regenerative intervention concepts utilizing a processed mVASC technology is explored: cases of complex wounds are treated in a minimally invasive manner (as an in-office procedure) with delivery of microvascular graft that supports tissue repair. The proposed method recognizes that the isolation and transplantation of nonviable microvascular fragments rapidly develop into bloodperfused microvascular networks after their transfer into tissue defects, changing the trajectory of healing from nonhealing to healing in a biologically hostile (avascular and fibrotic with cellular senescence) environment [43]. The recognition that microvascular tissue actively interacting with the recipient site may be a feasible starting source for a regenerative medicine-based platform therapy is explored and shows very encouraging results. The three wounds presented in this study were very severe wounds that would not be expected to heal with advanced biologics. In fact, the first two cases received multiple advanced biologic treatments without success before receiving mVASC.

These treatments exemplify how expression of factors such as cytokines, chemokines and growth factors that are essential for angiogenesis, cell recruitment and activation, multiplication and specialization restore healing capacity. mVASC, an allogeneic perivascular tissue technology consisting of these same biologically active elements but in a microvascular tissue matrix without viable cells, appears to lead to a similar effect in challenging and even hostile (e.g., fluid-exuding wound) healing environments.

\section{Conclusion}

Microvascular tissue can be carefully isolated, concentrated, lyophilized and radiation sterilized to preserve structural elements of native microvascular tissue and cell phenotypes, despite loss of cell viability and ability to proliferate. Numerous active cytokines, chemokines and growth factors are consistently present in mVASC cell and tissue fragments, and indicate that the biologic capability to broadly stimulate tissue repair and regeneration is wellpreserved in this lyophilized, sterilized microvascular tissue. mVASC also stimulated endothelial cell migration in vitro, increased the number and size of blood vessels that had grown into a Matrigel plug in a dose-dependent fashion, and led to significant revascularization in ischemic hindlimbs in SCID mice. These results support the capability of mVASC as a platform technology to repair, replace, reconstruct or supplement impaired microvasculature.

We have demonstrated as clinical proof-of-concept that mVASC can successfully stimulate closure of complex senescent and recalcitrant wounds as well as a pericardial effusion draining wound after treatment with the allogeneic mVASC, mVASC. Clinical trials of mVASC as a wound healing intervention are underway. The outcome of mVASC grafting illustrates how it can be used as an adjuvant in the management of difficult to heal wounds, change the 
biological milieu of the extremely fibrotic senescent cellular environment, restore vascularity and overall tissue quality and promote wound closure.

\section{Translational perspective}

Microvascular tissue biology lends itself to application as a technology for tissue repair and wound healing. The compositional elements form the basis for biological reconstruction of the tissue required for wound neovascularization, granulation, closure and remodeling. Combined with previously reported clinical use of mVASC in diabetic foot ulcers [44], clinical application of mVASC in three cases of problematic wounds demonstrate proof-of-concept for microvascular tissue technology. These translational preclinical results and clinical cases set the stage for the large randomized controlled clinical trials of mVASC now underway. Given the cost burden of wound care, there is a need for mechanistic studies to complement biological rationale for advanced wound healing modalities and real-world case studies along with robust clinical trials showing efficacy to justify their use [45]. To foster scientific transparency, the complete translational development of mVASC has been detailed in this single publication integrating basic science and clinical experience.

\section{Summary points}

- Microvascular tissue graft (mVASC) is a stable, sterile, off-the-shelf processed allogeneic mVASC product containing capillary structural elements and bioactive factors, including an array of active cytokines, chemokines and growth factors.

- mVASC stimulates vascular endothelial cell migration in vitro and blood vessel ingrowth in vivo in a dose-response manner.

- mVASC stimulates neovascularization leading to increased limb preservation in an in vivo ischemic hindlimb severe combined immunodeficiency mouse model.

- Clinical application of mVASC as a topical intervention in complex, recalcitrant or senescent wound environments has demonstrated its ability to stimulate durable wound closure, even in cases where other advanced biologic therapies failed to heal the wounds.

- These results suggest mVASC's microvascular structure serves as an extracellular matrix scaffold for revascularization while the microvascular fragments support angiogenesis and tissue signaling in the setting of compromised vascularity.

\section{Financial \& competing interests disclosure}

The authors declare the following financial interests: DR Peterson, R-H Mattern and DM Arm are employees of MicroVascular Tissues, Inc. (MVT); WW Li is a consultant to MVT. The authors have no other relevant affiliations or financial involvement with any organization or entity with a financial interest in or financial conflict with the subject matter or materials discussed in the manuscript apart from those disclosed.

No writing assistance was utilized in the production of this manuscript.

Ethical conduct of research

The patients in the presented cases provided written informed consent to receive mVASC. As mVASC is an HCT/P indicated for the repair, reconstruction, replacement or supplementation of microvascular tissue, no additional institutional approvals were required for its clinical use.

\section{Open access}

This work is licensed under the Attribution-NonCommercial-NoDerivatives 4.0 Unported License. To view a copy of this license, visit http://creativecommons.org/licenses/by-nc-nd/4.0/

\section{References}

1. Guo S, DiPietro LA. Factors affecting wound healing. J. Dent. Res. 89(3), 219-229 (2010).

2. Mathieu D, Linke J-C, Wattel F. Non-healing wounds. In: Handbook on Hyperbaric Medicine. Mathieu D (Ed.). Springer, Amsterdam, The Netherlands, p401-p427 (2006).

3. Menke NB, Ward KR, Witten TM, Bonchev DG, Diegelmann RF. Impaired wound healing. Clin. Dermatol. 25(1), 19-25 (2007).

4. Shaw TJ, Martin P. Wound repair at a glance. J. Cell Sci. 122(18), 3209-3213 (2009). 
5. Robson MC, Steed DL, Franz MG. Wound healing: biologic features and approaches to maximize healing trajectories. Curr. Probl. Surg. 38(2), 72-140 (2001).

6. Carter MJ, Fylling CP, Parnell LKS. Use of platelet rich plasma gel on wound healing: a systematic review and meta-analysis. Eplasty 11(e38), 382-410 (2011).

7. Ojeh N, Pastar I, Tomic-Canic M, Stojadinovic O. Stem cells in skin regeneration, wound healing, and their clinical applications. Int. J. Mol. Sci. 16(10), 25476-25501 (2015).

8. Karaaltin MV, Baghaki S. Adipose derived regenerative cell therapy for treating a diabetic wound: a case report. Wounds 24(1), e1-e5 (2012).

9. Akita S, Yoshimoto H, Ohtsuru A, Hirano A, Yamashita S. Autologous adipose-derived regenerative cells are effective for chronic intractable radiation injuries. Rad. Prot. Dosimetry 151(4), 656-660 (2012).

10. Konstantinow A, Arnold A, Djabali K et al. Therapy of ulcus cruris of venous and mixed venous arterial origin with autologous, adult, native progenitor cells from subcutaneous adipose tissue: a prospective clinical pilot study. J. Eur. Acad. Derm. Ven. 31(12), 2104-2118 (2017).

11. Vyas KS, Vasconez HC. Wounds healing: biologics, skin substitutes, biomembranes and scaffolds. Healthcare (Basel) 2(3), 356-400 (2014).

12. Granick M, Baetz NW, Labroo P, Milner S, Li WW. In vivo expansion and regeneration of full thickness functional skin with an autologous homologous skin construct: clinical proof of concept for chronic wound healing. Int. Wound J. 16(3), 841-846 (2019).

13. Kober J, Gugerell A, Schmid M et al. Wound healing effect of conditioned media obtained from adipose tissue on human cells: a comparative in vitro study. Ann. Plast. Surg. 77(2), 156-163 (2016).

14. Kogan S, Sood A, Granick M. Amniotic membrane adjuncts and clinical applications in wound healing: a review of the literature. Wounds 30(6), 168-173 (2018).

15. DiDomenico LA, Orgill DP, Galiano RD et al. Use of an aseptically processed, dehydrated human amnion and chorion membrane improves likelihood and rate of healing in chronic diabetic foot ulcers: a prospective, randomised, multi-centre clinical trial in 80 patients. Int. Wound J. 15(6), 950-957 (2018).

16. Gosain A, DiPietro LA. Aging and wound healing. World J. Surg. 28(3), 321-326 (2004).

17. Regulski M. Understanding diabetic induction of cellular senescence: a concise review. Wounds 30(4), 96-101 (2018).

18. Kimmel HW, Grant A, Ditata J. The presence of oxygen in wound healing. Wounds 28(8), 264-270 (2016).

19. Yuan SY, Rigor RR. Chapter 2: Structure and function of exchange microvessels. In: Regulation of Endothelial Barrier Function. Yuan YS, Rigor RR (Eds). Morgan \& Claypool Life Sciences, CA, USA (2010).

20. Xu J, Shi GP. Vascular wall extracellular matrix proteins and vascular diseases. Biochim. Biophys. Acta 1842(11), 2106-2119 (2014).

21. Secomb TW, Pries AR, Gaehtgens P. Chapter 9: Architecture and hemodynamics of microvascular networks. In: Biological Flow. Jaffrin YM, Caro GC (Eds). Springer Science+Business Media, NY, USA, p159-p176 (1995).

22. Bodnar RJ, Satish L, Yates CC, Wells A. Pericytes: a newly recognized player in wound healing. Wound Rep. Regen. 24(2), 204-214 (2016).

23. James AW, Zara JN, Corselli M et al. An abundant perivascular source of stem cells for bone tissue engineering. Stem Cells Trans. Med. 1(9), 673-684 (2012).

24. Adair TH, Montani J-P. Angiogenesis. Morgan \& Claypool Life Sciences, CA, USA (2010).

25. Tonnesen MG, Feng X, Clark RA. Angiogenesis in wound healing. J. Invest. Derm. Symp. Proc. 5(1), $40-46$ (2000).

26. Laschke MW, Menger MD. Adipose tissue-derived microvascular fragments: natural vascularization units for regenerative medicine. Trends Biotech. 33(8), 442-448 (2015).

27. Nunes SS, Krishnan L, Gerard CS et al. Angiogenic potential of microvessel fragments is independent of the tissue of origin and can be influenced by the cellular composition of the implants. Microcirculation 17(7), 557-567 (2010).

28. McDaniel JS, Pilia M, Ward CL, Pollot BE, Rathbone CR. Characterization and multilineage potential of cells derived from isolated microvascular fragments. J. Surg. Res. 192(1), 214-222 (2014).

29. Huang NF, Niiyama H, Peter $\mathrm{C}$ et al. Embryonic stem cell-derived endothelial cells engraft into the ischemic hindlimb and restore perfusion. Arterioscler. Thromb. Vasc. Biol. 30(5), 984-991 (2010).

30. Niiyama H, Huang NF, Rollins MD, Cooke JP. Murine model of hindlimb ischemia. J. Vis. Exp. 21(23), 1035 (2009).

31. Caplan AI, Correa D. The MSC: an injury drugstore. Cell Stem Cell 9(1), 11-15 (2011).

32. Chang CC, Krishnan L, Nunes SS et al. Determinants of microvascular network topologies in implanted neovasculatures. Arterioscler. Thromb. Vasc. Biol. 32(1), 5-14 (2012).

33. Kim Y, Ko H, Kwon IK, Shin K. Extracellular matrix revisited: roles in tissue engineering. Int. Neurourol. J. 20(Suppl. 1), S23-S29 (2016). 
34. Hayden MR, Sowers JR, Tyagi SC. The central role of vascular extracellular matrix and basement membrane remodeling in metabolic syndrome and type 2 diabetes: the matrix preloaded. Cardiovasc. Diabetol. 4, 9 (2005).

35. Li J, Zhang YP, Kirsner RS. Angiogenesis in wound repair: angiogenic growth factors and the extracellular matrix. Microsc. Res. Tech. 60(1), 107-114 (2003).

36. Eble JA, Niland S. The extracellular matrix of blood vessels. Curr. Pharm. Design. 15(12), 1385-1400 (2009).

37. Gimble JM, Frazier T, Wu X et al. A novel, sterilized microvascular tissue product improves healing in a murine pressure ulcer model. Plas. Recon. Surg. Glob. Open 6(11), e2010 (2018).

38. Campos AC, Groth AK, Branco AB. Assessment and nutritional aspects of wound healing. Curr. Opin. Clin. Nutr. Metab. Care 11(3), 281-288 (2008).

39. Broughton G 2nd, Janis JE, Attinger CE. The basic science of wound healing. Plast. Recon. Surg. 117(Suppl. 7), S12-S34 (2006).

40. Gaur M, Amaro-Ortiz A, Dobke M, Jordan I, Lunyak V. Acute genotoxic stress-induced senescence in human mesenchymal cells drives a unique composition of senescence messaging secretomes (SMS). J. Stem Cell Res. Ther. 7(8), 396-410 (2017)

41. Tchkonia T, Kirkland J. Aging, cell senescence, and chronic disease: emerging therapeutic strategies. JAMA 320(13), 1319-1320 (2018).

42. Szpalski C, Butala P, Vandegrift MT et al. Improving senescent wound healing with local and systemic therapies. Ann. Plast. Surg. 81(1), 96-105 (2018).

43. Demidova-Rice TN, Hamblin MR, Herman IM. Acute and impaired wound healing: pathophysiology and current methods for drug delivery, part 2: role of growth factors in normal and pathological wound healing: therapeutic potential and method of delivery. Adv. Skin Wound Care 25(8), 349-370 (2012).

44. Zelen CM, Gould LJ, Li WW. Clinical achievement of wound closure and tissue quality with a novel microvascular tissue graft. Wounds 31(4), E29-E32 (2019).

45. Leask F. Spotting "unproven" stem cell therapies in the wild. Biotechnqiues 67(6), 253-255 (2019). 